\title{
Does outstretching the arms improve postural stability?
}

Mitesh Patel $^{1}$, David Buckwell ${ }^{1}$, Malcolm Hawken ${ }^{2}$, Adolfo M. Bronstein ${ }^{1 *}$

${ }^{1}$ Division of Brain Sciences, Imperial College London, Charing Cross Hospital, London. W6 8RF, UK.

${ }^{2}$ School of Sport \& Exercise Sciences, Liverpool John Moores University, Liverpool, L3 3AF.

* Address for correspondence:

Professor Adolfo M Bronstein,

Division of Brain Sciences (Neuro-otology Unit),

Imperial College London,

Charing Cross Hospital,

London, W6 8RF

a.bronstein@imperial.ac.uk

\section{Highlights:}

- Tandem stance on a narrow beam was recorded with arms outstretched and to the side.

- Outstretching arms improves balance in tandem stance on a beam with eyes closed.

- The clinical test of tandem stance can be affected by arm position. 


\section{Abstract}

We spontaneously outstretch our arms when standing upon challenging surfaces, yet the effect of stretching the arms upon postural stability is unknown. We investigated whether stretching out the arms laterally improves postural control during tandem stance on a narrow beam. Twelve healthy participants stood upon a beam, right foot in front of the left foot, for 30 seconds with arms outstretched or down to the side, with eyes open and closed.

Mediolateral head movement was characterised by root mean square amplitude (RMS), sway path, velocity during the largest excursion and power spectrum. Spectra for lateral forces from a force platform beneath the beam were also recorded. Outstretching the arms significantly reduced RMS, sway path and velocity of maximum displacement of head movement with eyes closed but not with eyes open. A similar trend was present in the power spectra of head motion and sway platform lateral forces. In conclusion, outstretching the arms helps postural stability in challenging situations such as tandem stance on a narrow beam with eyes closed. Although the exact mechanisms require further investigation, the effects are most likely mediated by changes in segmental inertia and the ability to make corrective arm movements.

Keywords: Postural control; arms outstretched; balance; tandem stance; narrow beam 


\section{Introduction}

Standing upon a narrow, raised support increases postural unsteadiness. Intuitively, in such situations, we stretch the arms out and this presumably increases postural stability. However, studies investigating this directly are rare. A previous report in abstract form from our group suggested that postural control improved while standing upon a beam on foam if the arms were free to move [2]. Specifically, participants were instructed to either cross or to use their arms (FREE) in order to maintain balance upon the beam.

Similar to free arm movement, maintaining a stretched out arm position increases the height of the moment of inertia, which should theoretically improve postural control similar to the situation when a tightrope walker crosses a tightrope by holding a balancing pole.

To test the hypothesis that stretching out the arms increases postural stability, we have now undertaken a new study on the effects of stretching out the arms during tandem stance on a narrow beam (i.e., a narrow, raised support), a posture which is known to produce unsteadiness mainly in a mediolateral direction [4]. To examine the effect of varying task difficulty, participants performed the experiment with eyes open and eyes closed, given that stretching out the arms might contribute little unless the balancing task is considerably challenging, e.g. narrow beam and eyes closed.

\section{Materials and Methods}

\section{Participants}

Twelve self-reported healthy participants ( 5 females, 7 males; mean ages 28 and 27 years) took part, with ethical approval from the Imperial College Ethics Committee. 


\section{Equipment}

Mediolateral linear head position was measured using a Fastrak ${ }^{\mathrm{TM}}$ electromagnetic tracking system (Polhemus, VT, USA) mounted on a light-weight adjustable helmet. Mediolateral ground reaction forces were registered with a force platform (0R6-5-1, AMTI, MA, USA. 91 x $61 \times 17 \mathrm{~cm})$. A wooden beam $(110 \mathrm{~L}$ x $9.5 \mathrm{~W}$ x $5.2 \mathrm{H} \mathrm{cm})$ sat sagittally over the entire length of the force platform securely fitted to the platform to prevent it from moving. All data was sampled at $250 \mathrm{~Hz}$ (analogue-to-digital 16 bit converter) and recordings lasted 30 seconds.

\section{Procedure}

Participants stood on the beam $(22.2 \mathrm{~cm}$ above the ground (beam + force platform $))$ in tandem stance with the right foot in front of the left (all participants were right footed). Participants were instructed to stand with eyes either open or closed with arms either by the sides (AS) or outstretched laterally approximately at shoulder level (AO). The field of view encompassed a laboratory environment and participants faced a wall one metre away. This arrangement, with the visual background at short distance, was deliberate so that any differences between eyes open and eyes closed would be maximised. The four possible combinations of arm and vision conditions were allocated to each participant using a counterbalanced Latin Square design. There was a 60 second gap between recordings, when participants stood normally, and participants had 10 seconds to settle before recording started. 


\section{Analysis}

Three measures were calculated for mediolateral head movement after low-pass filtering ( 2 pole, 10Hz, Butterworth): 1) Root Mean Square Amplitude (RMS, cm), 2) Sway Path (SP, $\mathrm{mm}$ ) and 3) Velocity during the largest excursion (VEL, $\mathrm{cm} / \mathrm{s}$ ), that is, the mean head velocity during the largest amplitude head oscillation observed in the 30s recording (Figure 1D). These periods represent the maximum challenge to balance, namely when people may fall, in agreement with Hof et al. [3] who emphasised the importance of considering velocity (as momentum), in addition to displacement, when assessing stability.

Power spectra were calculated for mediolateral head movement and mediolateral forces over 0.06-10 Hz using Welch's method with 4096 points and a Hamming window [9]. Spectral power was separated into two bins between $0.06-0.7 \mathrm{~Hz}$ and $0.7 \mathrm{~Hz}-4 \mathrm{~Hz}$ for all conditions as Honegger et al. [4] reported a change in behaviour in tandem stance at around $0.7 \mathrm{~Hz}$, specifically they observed that movement of both the head and pelvis increased in relation to movement of the trunk above $0.7 \mathrm{~Hz}$.

\section{Statistics}

Data values were positively skewed so a natural log transformation was applied before performing a repeated-measures ANOVA for each head movement variable and for the power spectra with factors 'ARMS' (2 levels: arms outstretched, arms to the side) and 'VISION' (2 levels: eyes open, closed). Post-hoc paired $t$-tests were employed to examine individual 'ARMS' effect; $p$-values $<0.05$ were considered significant. Head sway data from 
one subject in the eyes open condition was absent. In this condition, data from 11 participants were analysed.

\section{Results}

Figures $1 \mathrm{~A}-\mathrm{C}$ show that the arms outstretched (AO) posture reduced mediolateral head sway during tandem stance with eyes closed; statistical details follow.

RMS amplitude of head movement showed a significant interaction between ARMS and VISION $[F=8.32, p=0.015]$. Post- hoc tests showed that AO (arms outstretched) reduced sway RMS values with eyes closed $(p=0.006)$ but not with eyes open. VEL also showed a significant interaction between ARMS and VISION [F=16.85, $\mathrm{p}=0.002]$. Post-hoc tests showed slower VEL with AO $(\mathrm{p}=0.040)$ with eyes closed but not eyes open. Given the previous results and the fact that the ARMS/VISION interaction for sway path approached significance as well [F=4.35, $\mathrm{p}=0.064]$, post-hoc tests were also performed. Post-hoc tests showed that AO reduced sway path with eyes closed $(p=0.042)$ but not open. Post-hoc tests for all three head movement measures were also conducted with the non-parametric Wilcoxon test and the results were the same.

Figure 2A shows power spectrum data for lateral head motion, in particular reduced power with arms outstretched and eyes closed. ANOVAs showed a significant ARMS/VISION interaction (0.06-0.7Hz: $F=34.26, p<0.001 ; 0.7-4 H z: F=38.92, p<0.001)$. Post-hoc tests showed reduced power spectra with AO with eyes closed between $0.06-0.7 \mathrm{~Hz}(\mathrm{p}<0.001)$ and $0.7-4 \mathrm{~Hz}(\mathrm{p}=0.001)$ but not with eyes open.

Figure 2B shows power spectrum of mediolateral forces. The ANOVAs showed a significant ARMS/VISION interaction (0.06-0.7Hz: $F=13.85, p=0.004 ; 0.7-4 H z: F=13.40, p=0.001)$. 
Post-hoc tests showed reduced power spectra with AO between $0.06-0.7 \mathrm{~Hz}$ with eyes closed $(\mathrm{p}=0.021)$. Perhaps surprisingly, with eyes open, larger power was found with AO between $0.06-0.7 \mathrm{~Hz}(\mathrm{p}<0.001)$ and $0.7-4 \mathrm{~Hz}(\mathrm{p}<0.001)$.

\section{Discussion}

We found a significant reduction of RMS mediolateral head displacement, sway path (which also implies mean sway velocity), velocity of maximum displacement (the velocity of the largest oscillation observed) and power spectra on outstretching the arms with eyes closed. In essence, this indicates that outstretching the arms improves postural balance, as expected.

With eyes closed participants can only use vestibular and proprioceptive information to control body movement and balance in relation to the external gravitational reference frame. The most likely explanation for the reduction in head movement lies in the mechanical effects: the centre of gravity of the whole upper body (head, trunk and arms, HAT) is raised by about 50 millimetres, and its moment of inertia about its $\mathrm{CoM}$ is increased by about $70 \%$ (representative values estimated from data in Zatsiorsky [10]). Further, active movement of the arms relative to the trunk can generate restoring torques to aid balancing (see Figure 2 in Honegger et al. [4] for an example in tandem standing on foam).

The reduction in head movement by itself indicates better control of balance, without implying any particular model of balance strategy. However, this reduction was not observed with eyes open. This may be due to a 'ceiling effect', namely that control of posture with eyes open whilst seeing a target $1 \mathrm{~m}$ away was already close to optimal [7] and therefore stretching the arms does not afford any noticeable improvement. Honegger et al. [4] provide supporting evidence for this latter concept in that their participants voluntarily adopted the 
arms down posture in this condition, only outstretching the arms when conditions became more difficult. Also, arm movement (hands on hips) contributed less than $10 \%$ of the variance in tandem stance with eyes open in the study by Federolf et al. [1]. The increased spectral power in the force data with eyes open could reflect the larger forces generated [10]) to control the increased inertia and higher $\mathrm{COM}$ in the $\mathrm{AO}$ condition.

One clinical implication of our results, along with the findings of [6] and [8], is that the test of tandem stance would be affected by arm position and movement, so specific instructions to patients would be appropriate. Often, patients with balance difficulty stretch out their arms when they are about to lose balance [5]. In addition to precaution, our findings suggest that this behaviour could help to avoid a stumble.

\section{Acknowledgements}

This work was supported by an MRC grant to A.M.B. (MC_U950770497). M.P. is funded by the Ménière's Society, UK.

\section{References}

[1] P. Federolf, L. Roos, B.M. Nigg, Analysis of the multi-segmental postural movement strategies utilized in bipedal, tandem and one-leg stance as quantified by a principal component decomposition of marker coordinates, Journal of Biomechanics 46 (2013) 26262633.

[2] D.A. Green, Bronstein, A. M., Balance task adaptation with and without arm movement, Proceedings of The Physiological Society 565P (2005).

[3] A.L. Hof, M.G. Gazendam, W.E. Sinke, The condition for dynamic stability, Journal of biomechanics 38 (2005) 1-8.

[4] F. Honegger, R.J. Tielkens, J.H. Allum, Movement strategies and sensory reweighting in tandem stance: differences between trained tightrope walkers and untrained subjects, Neuroscience 254 (2013) 285-300.

[5] P. Meyns, K. Desloovere, L. Van Gestel, F. Massaad, B. Smits-Engelsman, J. Duysens, Altered arm posture in children with cerebral palsy is related to instability during walking, European journal of paediatric neurology : EJPN : official journal of the European Paediatric Neurology Society 16 (2012) 528-535.

[6] M. Milosevic, K.M.V. McConville, K. Masani, Arm movement improves performance in clinical balance and mobility tests, Gait Posture 33 (2011) 507-509.

[7] W. Paulus, A. Straube, S. Krafczyk, T. Brandt, Differential effects of retinal target displacement, changing size and changing disparity in the control of anterior/posterior and 
lateral body sway, Experimental brain research. Experimentelle Hirnforschung. Experimentation cerebrale 78 (1989) 243-252.

[8] M. Shafeie, S. Manifar, M. Milosevic, K.M.V. McConville, Arm movement effect on balance. Engineering in Medicine and Biology Society (EMBC), 2012 Annual International Conference of the IEEE, 2012, pp. 4549-4552.

[9] K. Yarrow, P. Brown, M.A. Gresty, A.M. Bronstein, Force platform recordings in the diagnosis of primary orthostatic tremor, Gait \& posture 13 (2001) 27-34.

[10] V.M. Zatsiorsky, Kinetics of Human Motion, Human Kinetics, Champain, IL, USA, 2002, 653 pp.

\section{Figure Legends}

Figure 1: Plots of mean and SEM of natural log transformed Root Mean Square (A), Sway Path (B) and Velocity during the largest excursion (C) of mediolateral head movement during tandem stance on a narrow beam. All measures of mediolateral head movement were reduced by extending the arms with eyes closed, but not with eyes open. Asterisks are post-hoc differences $\left(* * \mathrm{p}<0.01 \&{ }^{*} \mathrm{p}<0.05\right)$. Mediolateral head motion from a single subject is shown in (D). Mean velocity during the largest excursion was attained by the slope visually fitted to the largest amplitude of head oscillation.

Figure 2: Log mean $(n=12)$ energy spectra of mediolateral head movement $(A)$ and mediolateral ground reaction forces (B) during tandem stance on a narrow beam. Statistics are shown for bins between $0.06-0.7 \mathrm{~Hz}$ and $0.7-4 \mathrm{~Hz}$ comparing Arms Outstretched to Arms to the Side. ${ }^{* *} \mathrm{p}<0.001$, ${ }^{*} \mathrm{p}<0.01,{ }^{*} \mathrm{p}<0.05$ and NS $=$ Not-Significant. 


\section{Figure 1}

A. Ln Root Mean Square of mediolateral head movement

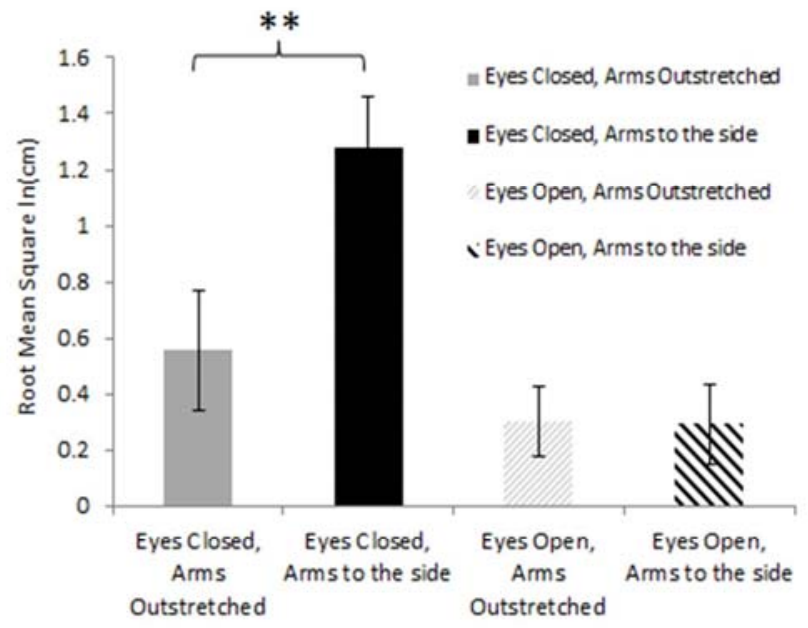

C. Ln Velocity of mediolateral maximum displacement

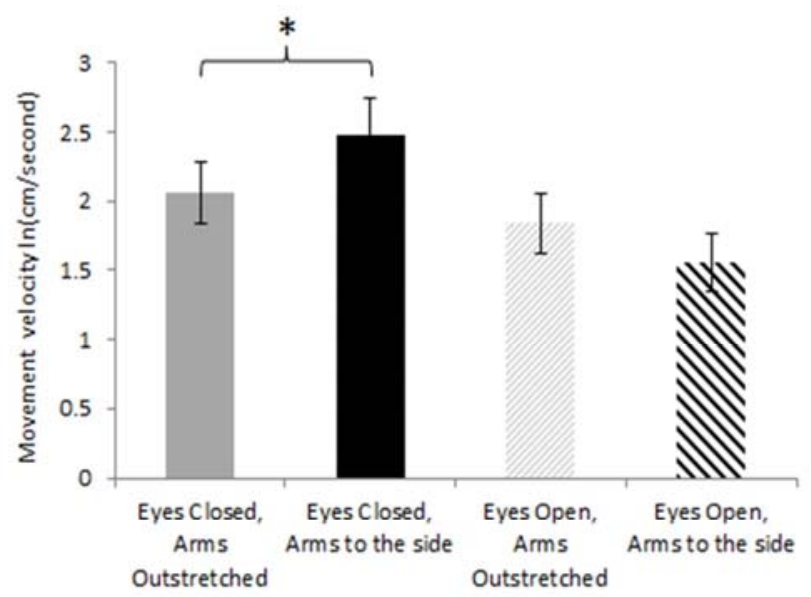

B. Ln Sway path of mediolateral head movement

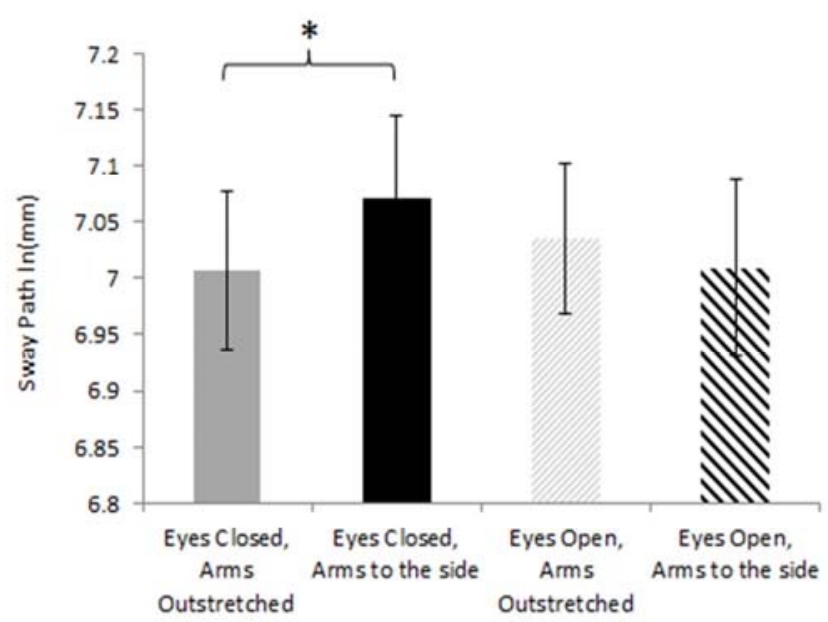

D. Mediolateral head movement showing velocity during the largest excursion

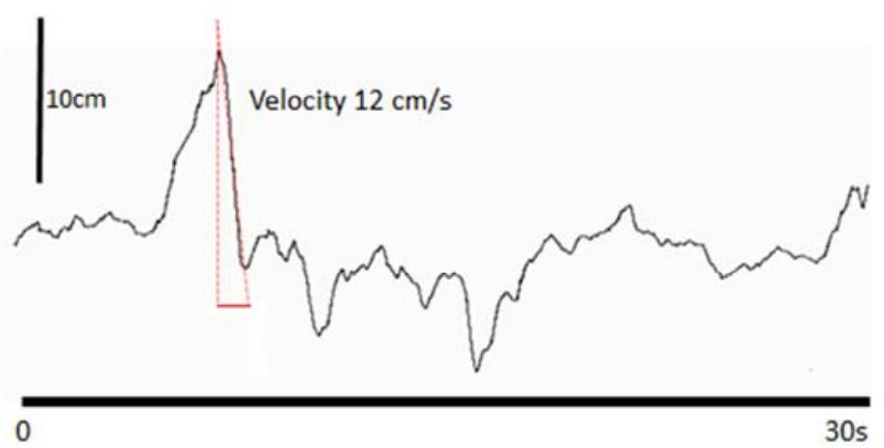


Figure 2 
A. Fast Fourier transform log mediolateral head movement

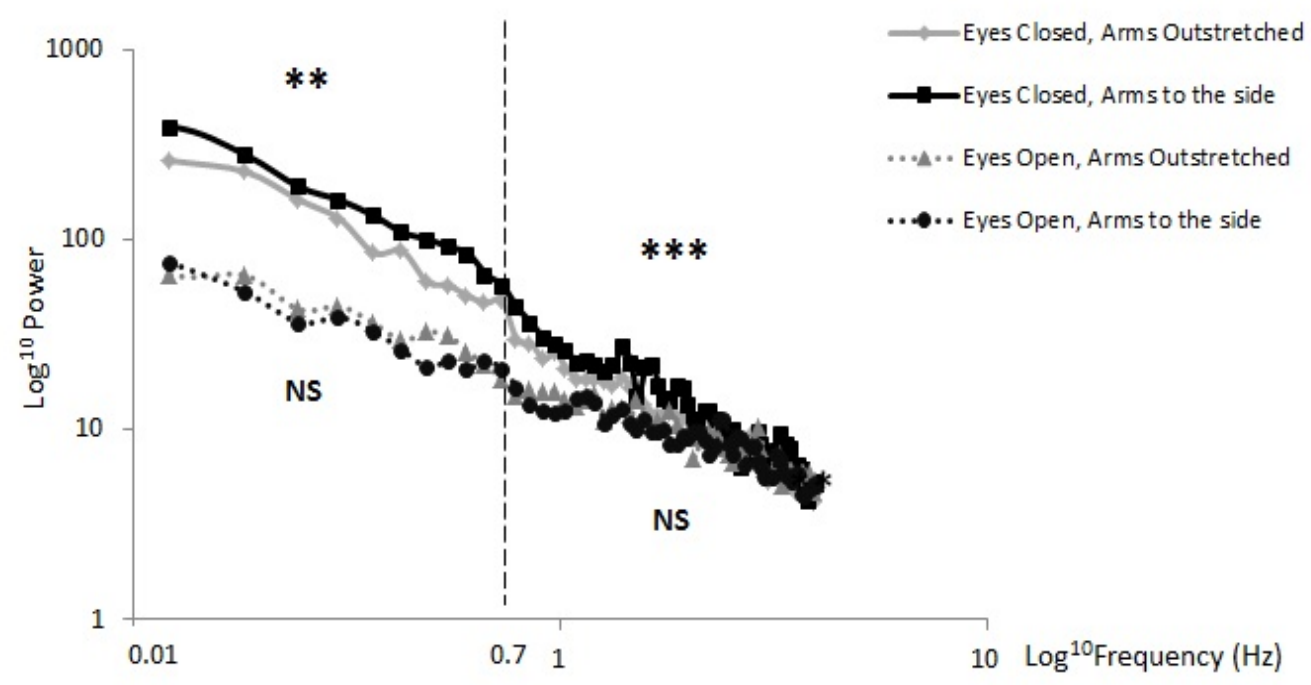

B. Fast Fourier transform log mediolateral forces

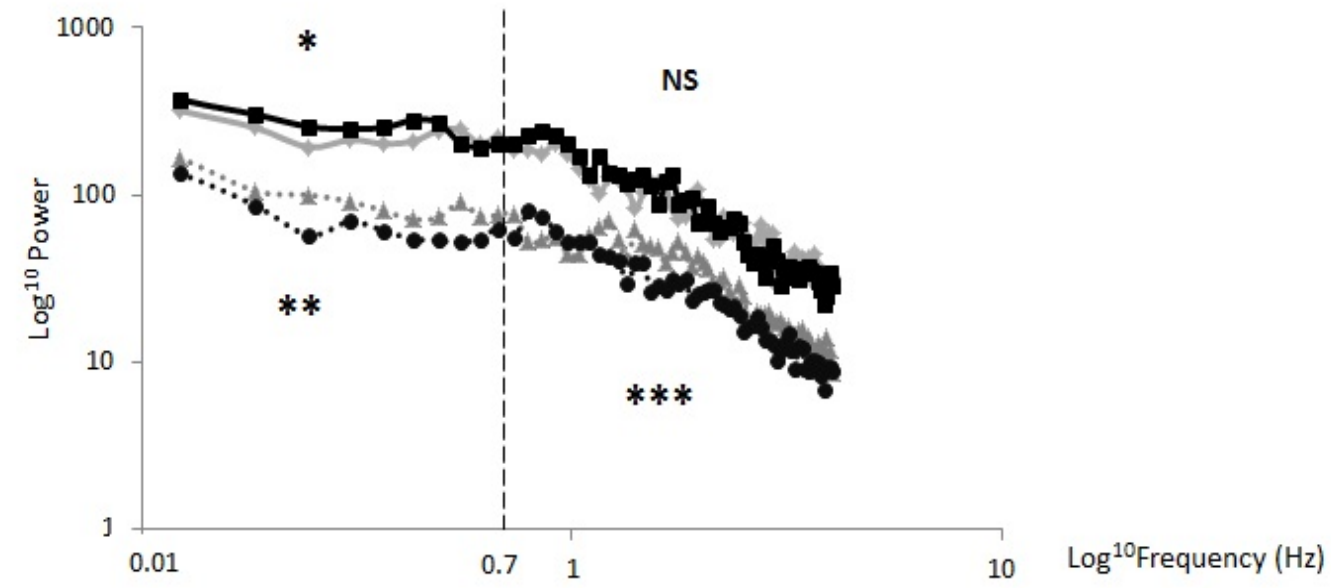

